\title{
Diversidade genética de populações de andiroba no Baixo Acre
}

\author{
Andréa Raposo ${ }^{(1)}$, Karina Martins ${ }^{(2)}$, Ana Yamaguishi Ciampi( ${ }^{(3)}$, Lúcia Helena de Oliveira Wadt ${ }^{(2)}$ \\ e Elizabeth Ann Veasey ${ }^{(1)}$
}

\begin{abstract}
(1)Escola Superior de Agricultura Luiz de Queiroz, Dep. de Genética, Av. Pádua Dias, no 11, CEP 13400-970 Piracicaba, SP. E-mail: andrearaposo@gmail.com, eaveasey@esalq.usp.br (2)Embrapa Acre, Rod. BR 364, Km 14, Caixa Postal 321, CEP 69908-970 Rio Branco, AC. E-mail: karimartins@yahoo.com, lucia@cpafac.embrapa.br (3)Embrapa Recursos Genéticos e Biotecnologia, PqEB, Av. W5 Norte (final), Caixa Postal 02372, CEP 70770-900 Brasília, DF. E-mail: aciampi@cenargen.embrapa.br
\end{abstract}

\begin{abstract}
Resumo - O objetivo deste trabalho foi avaliar a diversidade genética em duas populações de Carapa guianensis Aubl. (andiroba), no Estado do Acre, e comparar os parâmetros de diversidade com os observados em outras populações da espécie (no Brasil: Flona Tapajós, PA, Porto Acre, AC; e na Costa Rica). Foram avaliados 77 indivíduos adultos com sete locos polimórficos de microssatélites. Observaram-se 51 alelos nas duas populações, em que o número efetivo de alelos por loco $\left(\hat{\mathrm{A}}_{\mathrm{e}}=3,2\right)$ foi inferior ao número médio de alelos por loco ( $\hat{A}=7,3)$, o que indica elevado número de alelos com baixa frequiência. Os valores estimados de f̂ não diferiram de zero, o que mostra que não ocorre endogamia nas populações. A taxa de cruzamento aparente foi alta $\left(\hat{t}_{a}=1,11\right.$ na população Porto Acre, e $\hat{t}_{a}=0,88$ na de Rio Branco), resultado indicador de que a espécie se reproduz por alogamia. Foi observado, por meio das estimativas de $\hat{A}, \hat{H}_{\mathrm{e}}$ (diversidade gênica) e $\hat{\mathrm{N}}_{\mathrm{e}}$ (número efetivo populacional) que as populações de andiroba, comparadas neste trabalho, tiveram padrões de diversidade semelhantes, porém, proporções de alelos raros diferentes.
\end{abstract}

Termos para indexação: Carapa guianensis, marcadores microssatélites, tamanho efetivo populacional, conservação de recursos genéticos, alelos raros, fluxo gênico.

\section{Genetic diversity structure of crabwood in Baixo Acre, Brazil}

\begin{abstract}
The objective of this work was to evaluate the genetic diversity of two Carapa guianensis Aubl. (crabwood) populations, in the State of Acre, Brazil, and to compare the diversity estimations with those obtained in other populations of the species (in Brazil: Flona Tapajós, PA, Porto Acre, AC; and in Costa Rica). Seventyseven individuals were assessed using seven polymorphic microsatellite loci. Fifty-one alleles were observed in the two populations, in which the effective number of alleles per locus $\left(\hat{\mathrm{A}}_{\mathrm{e}}=3.2\right)$ was lower than the average number of alleles per locus $(\hat{A}=7.3)$, which indicates a high number of low frequency alleles. The estimated $\hat{f}$ values did not differ from zero, showing that inbreeding does not occur in these populations. The apparent outcrossing rate was high for both populations $\left(\hat{t}_{a}=1.11\right.$ in Porto Acre population, and $\hat{t}_{a}=0.88$ in Rio Branco), indicating that this species reproduces by outcrossing. Comparing the estimates of $\hat{\mathrm{A}}_{\mathrm{e}}, \hat{\mathrm{H}}_{\mathrm{e}}$ (gene diversity) and $\hat{\mathrm{N}}_{\mathrm{e}}$ (effective population number), it was observed that the general patterns of genetic diversity were similar. However, the number of rare alleles differ between populations.
\end{abstract}

Index terms: Carapa guianensis, microsatellite markers, effective population size, genetic resources conservation, rare alleles, gene flow.

\section{Introdução}

O entendimento dos padrões de variação genética, entre e dentro de populações, é de fundamental importância para o desenvolvimento de estratégias de conservação, tanto para definir unidades apropriadas para conservação in situ, como para desenvolver estratégias efetivas de coleta de sementes para conservação ex situ (Hamrick \& Godt, 1996).
A exploração predatória de espécies arbóreas, nas florestas tropicais, pode promover uma série de alterações, tais como: redução no tamanho efetivo populacional, mudanças no padrão de distribuição espacial, no comportamento de polinizadores e dispersores de sementes, na taxa de cruzamento, entre outras (Loveless \& Hamrick, 1984; Lande, 1988; Young et al., 1996). Todos estes fatores podem afetar a estrutura genética das populações e, como 
consequiências, trazer a perda de alelos raros, a redução da heterozigosidade e o aumento da endogamia. A perda de alelos leva à redução, na capacidade das espécies, de responder a adversidades ambientais em gerações futuras; pode ocorrer redução na adaptabilidade das espécies (Young et al., 1996). Portanto, a caracterização dos graus de variabilidade e estrutura genética e o conhecimento da movimentação de alelos proporcionam as bases necessárias da execução de estratégias, para maximizar a eficiência dos programas de manejo e conservação genética.

Os parâmetros mais utilizados para estimar a diversidade genética em populações naturais e as alterações decorrentes da ação antrópica, empregandose marcadores altamente polimórficos como os microssatélites ou SSR ("simple sequence repeats"), são: número médio de alelos por loco (Â), heterozigosidade esperada ou diversidade gênica $\left(\hat{\mathrm{H}}_{\mathrm{e}}\right)$; heterozigosidade observada $\left(\hat{\mathrm{H}}_{\mathrm{o}}\right)$ e índice de fixação $(\hat{\mathrm{f}})$ (Berg \& Hamrick, 1997). Entre estes parâmetros, $\hat{A}$ e $\hat{H}_{\mathrm{e}}$ são muito influenciados pela deriva genética, em razão de os alelos raros (com freqüência inferior a 0,05 ) não serem retidos em pequenas amostras. Porém, para inferir se a diversidade genética em uma população será mantida em longo prazo, apenas a quantificação da riqueza alélica (Â) é insuficiente. Para esse tipo de inferência, é necessária a análise comparativa de $\hat{\mathrm{A}} \mathrm{e} \hat{\mathrm{H}}_{\mathrm{e}}$, especialmente porque o valor de $\hat{\mathrm{H}}_{\mathrm{e}}$ é influenciado tanto pelo número de alelos como pela distribuição de suas frequiências relativas.

Um outro parâmetro muito utilizado nas atividades de preservação de germoplasma, coleta de sementes e conservação in situ é o tamanho efetivo populacional $\left(\hat{\mathrm{N}}_{\mathrm{e}}\right)$, que depende do número de indivíduos que efetivamente participam da reprodução e de sua contribuição relativa para a geração seguinte (Crossa \& Vencovsky, 1994). É, portanto, uma medida de representatividade genética, sendo que quanto maior o $\hat{\mathrm{N}}_{\mathrm{e}}$ menor a magnitude da deriva genética e, da mesma forma, quanto maior sua magnitude menor será o efeito da endogamia na população (Loveless \& Hamrick, 1984). Na prática, o $\hat{\mathrm{N}}_{\mathrm{e}}$ é estimado com base no tamanho amostral e no índice de fixação, duas medidas indiretas da sensibilidade da população à perda de alelos por deriva genética.

A andiroba é uma espécie arbórea clímax, de crescimento rápido, que ocorre em toda a Região Amazônica. Apresenta floração assincrônica e prolongada, e é polinizada por insetos de pouca mobilidade como microlepidópteros e meliponinas (Maués, 2006), é auto-incompatível (Hall et al., 1994; Maués, 2006) e tem elevada densidade populacional nas áreas de ocorrência.

O objetivo deste trabalho foi avaliar a diversidade genética em duas populações de Carapa guianensis Aubl. (andiroba), no Estado do Acre, e comparar os parâmetros de diversidade com os observados em outras populações da espécie (no Brazil: Flona Tapajós, PA, Porto Acre, AC; e na Costa Rica).

\section{Material e Métodos}

Foram avaliadas duas populações de andiroba (C. guianensis) no Estado do Acre, Uma localizada no Projeto de Assentamento Extrativista Caquetá (9³7'29"S e $\left.67^{\circ} 27^{\prime} 25^{\prime \prime W}\right)$, no Município de Porto Acre, e a outra na Reserva Florestal da Embrapa Acre $\left(10^{\circ} 1\right.$ '28"S e 67²'19"W), no Município de Rio Branco. A distância geográfica entre as duas regiões é de aproximadamente $71 \mathrm{~km}$. Amostras foliares de 39 indivíduos adultos reprodutivos da população de andiroba de Porto Acre e 38 da população de Rio Branco foram enviadas para o Laboratório de Genética Vegetal da Embrapa Recursos Genéticos e Biotecnologia, em Brasília, onde foram realizadas as análises genéticas.

A extração do DNA genômico foi realizada com o tampão CTAB 2\% (Doyle \& Doyle, 1990). Após a quantificação, o DNA das amostras foi diluído em água MilliQ a $1 \mathrm{ng} \mu \mathrm{L}^{-1}$. Foram utilizados sete pares de iniciadores específicos para Carapa guianensis, cinco desenvolvidos por Vinson et al. (2005) (Cg 01, Cg 06, Cg 12, Cg 16 e Cg 17), e dois por Dayanandan et al. (1999) (Cg 05 e Cg 07, aqui nomeados de Cg 05p e Cg 07p, respectivamente). O iniciador "forward" de cada loco foi marcado com um fluorocromo específico. As reações de PCR foram otimizadas para $6 \mu \mathrm{L}$ de volume final, com 0,33 ng de DNA genômico; $1 \mathrm{X}$ de tampão de reação da PCR (10 mM Tris- $\mathrm{HCl}, \mathrm{pH} 8,3$, $50 \mathrm{mM}$ de $\mathrm{KCl}, 1,5 \mathrm{mM}$ de $\mathrm{MgCl}_{2}$, $\mathrm{pH} 8,3$; Phoneutria); $0,25 \mu \mathrm{M}$ de cada iniciador ("forward" marcado e "reverse"); 0,27 $\mathrm{mg} \mathrm{mL}^{-1}$ de BSA (albumina sérica bovina); 0,27 mM de dNTP e 0,125 U Taq polimerase (Phoneutria) e água MilliQ estéril. As amplificações foram realizadas nos termocicladores 9600 e 9700 , nas seguintes condições: $94^{\circ} \mathrm{C}$ por $5 \mathrm{~min} ; 30$ ciclos de $94^{\circ} \mathrm{C}$ por $1 \mathrm{~min}$; temperatura de hibridação específica para cada par de iniciadores (Tabela 1) por $1 \mathrm{~min} ; 72^{\circ} \mathrm{C}$ por $1 \mathrm{~min}$; e finalização com $72^{\circ} \mathrm{C}$ por $7 \mathrm{~min}$. 
O produto das reações foi diluído na proporção $1: 3(1 \mu \mathrm{L}$ de reação para $3 \mu \mathrm{L}$ de água MilliQ). Em seguida, $1 \mu \mathrm{L}$ dessa diluição foi colocado em placa de microtitulação de 96 poços, que continha $9 \mu \mathrm{L}$ de formamida $\mathrm{HiDi}, 0,25 \mu \mathrm{L}$ de marcador interno fluorescente (ROX) (Brondani \& Gratapaglia, 2001) e $0,75 \mu \mathrm{L}$ de água MilliQ. Após a desnaturação $\left(95^{\circ} \mathrm{C}\right.$ por $3 \mathrm{~min}$ ), a placa foi levada para o seqüenciador $\mathrm{ABI} 3700$, onde ocorreu a eletroforese capilar. A detecção e a estimativa do tamanho dos alelos, em pares de base, foram realizadas com o uso dos programas GeneScan (Applied Biosystems, 2001) e Genotyper (Applied Biosystems, 1996).

Para cada loco, as estimativas do tamanho dos alelos em pares de base foram determinadas como sendo par ou ímpar de acordo com o tamanho previsto no desenvolvimento do marcador. Alelos com tamanho estimado intermediário às classes esperadas foram definidos como alelos da classe mais próxima (inferior ou superior) com maior frequiência. Para evitar a superestimativa da quantidade de alelos, além da estratégia supracitada, visualizaram-se os alelos em histogramas no programa Genotyper, e observou-se a sobreposição de todos os alelos da mesma placa de PCR.

As frequiências alélicas foram estimadas para os indivíduos das duas populações com o uso do programa

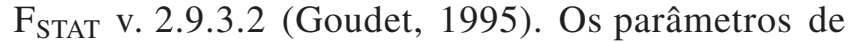
diversidade genética intrapopulacional foram estimados com uso do programa GDA (Lewis \& Zaykin, 2002): número médio de alelos por loco (Â); diversidade gênica $\left(\hat{H}_{e}\right)$ em equilíbrio de Hardy-Weinberg; heterozigosidade observada $\left(\hat{\mathrm{H}}_{\mathrm{o}}\right)$; e índice de fixação $(\hat{\mathrm{f}})$, o qual foi estimado de acordo com Weir (1996): $\hat{f}=1-\hat{H}_{0} / \hat{H}_{e}$.

Para verificar se os valores médios de f eram diferentes de zero, estimou-se o intervalo de confiança a 99\% de probabilidade, pelo método de boostrap, com 10 mil reamostragens sobre os locos pelo programa GDA (Lewis \& Zaykin, 2002).
A máxima diversidade possível, em cada loco, foi calculada a partir do número de alelos observados, em que: $\mathrm{H}_{\max }=(\hat{\mathrm{A}}-1) / \hat{\mathrm{A}}$. O número efetivo de alelos por loco $\left(\hat{\mathrm{A}}_{\mathrm{e}}\right)$ foi calculado a partir de $\hat{\mathrm{A}}_{\mathrm{e}}=1 /\left(1-\hat{\mathrm{H}}_{\mathrm{e}}\right)$. A taxa de cruzamento aparente foi estimada conforme Weir (1996) e Vencovsky (1994), em que $\hat{t}_{a}=(1-\hat{f}) /(1+\hat{f})$. O tamanho populacional efetivo $\left(\hat{N}_{\mathrm{e}}\right)$ foi estimado de acordo com Vencovsky \& Crossa (2003), em que $\hat{\mathrm{N}}_{\mathrm{e}}=\mathrm{n} /(1+\hat{\mathrm{f}})$. Foi verificada a representatividade genética $\left(\hat{\mathrm{N}}_{\mathrm{e}} / \mathrm{N}\right)$ das amostras. As estimativas da diversidade genética foram comparadas com outros estudos, com a mesma espécie, realizados no Brasil (Porto Acre, AC e Flona Tapajós, PA) e na Costa Rica, com uso de marcadores SSR. Foram obtidas estimativas de $\hat{\mathrm{H}}_{\max }$ e $\hat{\mathrm{N}}_{\mathrm{e}}$ para essas populações, com base nos resultados de $\hat{\mathrm{A}}, \hat{\mathrm{H}}_{\mathrm{e}}$ e $\hat{\mathrm{f}}$ obtidos por Dayanandan et al. (1999), Kageyama et al. (2004) e Cloutier et al. (2007).

Estimou-se, ainda, a divergência genética entre populações ( $\mathrm{R}_{\mathrm{ST}}$ ) (Slatkin, 1995), com o auxílio do programa RSTCALC v.2.2 (Goodman, 1997). O intervalo de confiança a $95 \%$ de probabilidade foi obtido pelo método de reamostragem bootstrap, com uso de 10 mil repetições sobre os locos. O fluxo gênico aparente $(\mathrm{Nm})$, entre as populações, foi estimado pelo método indireto, calculado de acordo com o modelo proposto por Wright (1951), segundo a fórmula: $\mathrm{Nm}=1 / 4\left[\left(1 / \hat{\mathrm{F}}_{\mathrm{ST}}\right)-1\right] . \mathrm{O} \hat{\mathrm{F}}_{\mathrm{ST}}$ foi substituído pelo $\mathrm{R}_{\mathrm{ST}}$, para uma estimação não viesada do fluxo gênico.

\section{Resultados e Discussão}

Ambas as populações avaliadas apresentaram altos índices de diversidade genética. Os sete locos SSR polimórficos utilizados permitiram observar 51 alelos, em 77 indivíduos das duas populações. Os locos mais polimórficos foram o Cg 16 e o Cg 5p, com 11 alelos cada, e o loco $\mathrm{Cg} 01$ apresentou menor polimorfismo, com apenas três alelos observados (Tabela 2).

Tabela 1. Estimativas de parâmetros genéticos, em sete locos de Carapa guianensis, em que $\mathrm{T}_{\mathrm{a}}$ é a temperatura de hibridação; n é o número de indivíduos analisados; $\hat{A}$ é o número de alelos por loco; $\hat{\mathrm{A}}_{\mathrm{e}}$ é o número efetivo de alelos, por loco; $\hat{\mathrm{H}}_{\mathrm{o}}$ é a heterozigosidade observada; $\hat{H}_{\mathrm{e}}$ é a diversidade gênica; $\hat{\mathrm{H}}_{\max }$ é a máxima diversidade para cada loco; $\hat{\mathrm{H}}_{\mathrm{e}} / \hat{\mathrm{H}}_{\max }$ é a proporção da diversidade máxima.

\begin{tabular}{|c|c|c|c|c|c|c|c|c|}
\hline Locos & $\mathrm{T}_{\mathrm{a}}\left({ }^{\circ} \mathrm{C}\right)$ & $\mathrm{n}$ & $\hat{\mathrm{A}}$ & $\hat{A}_{e}$ & $\hat{\mathrm{H}}_{\mathrm{o}}$ & $\hat{\mathrm{H}}_{\mathrm{e}}$ & $\hat{\mathrm{H}}_{\max }$ & $\hat{\mathrm{H}}_{\mathrm{e}} / \hat{\mathrm{H}}_{\max }(\%)$ \\
\hline $\mathrm{Cg} 01$ & 56 & 77 & 3 & 1,250 & 0,195 & 0,200 & 0,667 & 30,0 \\
\hline $\mathrm{Cg} 06$ & 56 & 77 & 5 & 1,859 & 0,389 & 0,462 & 0,800 & 57,7 \\
\hline $\mathrm{Cg} 12$ & 52 & 76 & 6 & 2,577 & 0,789 & 0,612 & 0,833 & 73,4 \\
\hline $\mathrm{Cg} 16$ & 56 & 77 & 11 & 6,168 & 0,792 & 0,838 & 0,909 & 92,2 \\
\hline Cg 17 & 52 & 77 & 7 & 3,413 & 0,675 & 0,707 & 0,857 & 82,5 \\
\hline $\mathrm{Cg} 05 \mathrm{p}$ & 56 & 77 & 11 & 4,017 & 0,636 & 0,751 & 0,909 & 82,6 \\
\hline $\mathrm{Cg} 07 \mathrm{p}$ & 52 & 77 & 8 & 3,030 & 0,571 & 0,670 & 0,875 & 76,6 \\
\hline Média & & 76,857 & 7,3 & 3,188 & 0,578 & 0,606 & 0,836 & 70,7 \\
\hline
\end{tabular}


Considerando-se as duas populações, o número médio de alelos por loco foi $\hat{A}=7,3$ (Tabela 1). Essa estimativa

Tabela 2. Frequiência dos alelos observados em cada loco nas populações de Carapa guianensis, em Porto Acre (39 indivíduos adultos reprodutivos) e em Rio Branco (38 indivíduos adultos reprodutivos).

\begin{tabular}{|c|c|c|c|}
\hline Locos & Alelos & Porto Acre & Rio Branco \\
\hline \multirow[t]{3}{*}{$\mathrm{Cg} 01$} & 198 & 0,013 & 0,013 \\
\hline & 200 & 0,936 & 0,842 \\
\hline & 204 & 0,051 & 0,145 \\
\hline \multirow[t]{5}{*}{$\mathrm{Cg} 06$} & 140 & 0,462 & 0,145 \\
\hline & 142 & 0,487 & 0,855 \\
\hline & 150 & 0,013 & - \\
\hline & 154 & 0,026 & - \\
\hline & 156 & 0,013 & - \\
\hline \multirow[t]{6}{*}{$\mathrm{Cg} 12$} & 143 & 0,013 & - \\
\hline & 147 & 0,145 & - \\
\hline & 151 & 0,026 & 0,039 \\
\hline & 153 & 0,211 & 0,342 \\
\hline & 155 & - & 0,118 \\
\hline & 157 & 0,605 & 0,500 \\
\hline \multirow[t]{11}{*}{$\mathrm{Cg} 16$} & 151 & 0,026 & - \\
\hline & 159 & 0,308 & 0,053 \\
\hline & 161 & 0,013 & - \\
\hline & 163 & 0,269 & 0,184 \\
\hline & 165 & 0,192 & 0,224 \\
\hline & 167 & 0,128 & 0,132 \\
\hline & 169 & 0,038 & 0,237 \\
\hline & 171 & 0,026 & 0,092 \\
\hline & 173 & - & 0,026 \\
\hline & 175 & - & 0,039 \\
\hline & 179 & - & 0,013 \\
\hline \multirow[t]{7}{*}{$\operatorname{Cg} 17$} & 203 & 0,013 & - \\
\hline & 207 & 0,141 & - \\
\hline & 211 & 0,051 & 0,092 \\
\hline & 213 & 0,333 & 0,539 \\
\hline & 215 & 0,282 & 0,303 \\
\hline & 217 & 0,154 & 0,066 \\
\hline & 219 & 0,026 & - \\
\hline \multirow[t]{11}{*}{ Cg 05p } & 170 & - & 0,013 \\
\hline & 172 & - & 0,132 \\
\hline & 174 & 0,359 & 0,303 \\
\hline & 176 & 0,410 & 0,303 \\
\hline & 178 & 0,038 & 0,026 \\
\hline & 180 & 0,013 & 0,118 \\
\hline & 184 & 0,051 & 0,066 \\
\hline & 190 & - & 0,026 \\
\hline & 192 & 0,115 & - \\
\hline & 194 & 0,013 & - \\
\hline & 204 & - & 0,013 \\
\hline \multirow[t]{8}{*}{$\mathrm{Cg} \mathrm{07p}$} & 214 & 0,051 & - \\
\hline & 216 & 0,462 & 0,526 \\
\hline & 218 & 0,013 & 0,013 \\
\hline & 220 & 0,449 & 0,079 \\
\hline & 226 & 0,026 & 0,092 \\
\hline & 228 & - & 0,026 \\
\hline & 232 & - & 0,250 \\
\hline & 234 & - & 0,013 \\
\hline
\end{tabular}

foi semelhante à observada em outros estudos com essa espécie (Tabela 3), apesar de o número amostral deste trabalho ter sido significativamente inferior aos demais trabalhos.

A população Porto Acre apresentou dois alelos a mais que a Rio Branco, e não se observou variação no número médio de alelos por loco e no número efetivo de alelos por loco (Tabela 4), entre as populações. Foram observados 13 alelos exclusivos na população Porto Acre e 11 na Rio Branco, e a maioria desses ocorreu em freqüências muito baixas, de uma a duas vezes nas populações. Isso indica que, para a maioria deles, o fato de serem exclusivos foi decorrente da baixa frequiência. Entretanto, ressalta-se a presença de alguns alelos exclusivos, com freqüências acima de 0,1 (10\%), nas duas populações (Tabela 2). Observaram-se 17 alelos raros, na população Porto Acre e 12 na população Rio Branco (Tabela 2), ou seja, 42,5\% de alelos com frequiência inferior a 0,05 na população Porto Acre e $31,6 \%$ na população Rio Branco.

Tanto para a população Porto Acre, quanto para a Rio Branco, as estimativas de $\hat{\mathrm{H}}_{\mathrm{e}}$ e $\hat{\mathrm{H}}_{\mathrm{o}}$ foram semelhantes (já que os erros-padrão se sobrepõem) e, como conseqüência, os valores de $\hat{\mathrm{f}}$ não diferiram de zero (Tabela 4). Estes dados são concordantes com os de Dayanandan et al. (1999), em populações de C. guianensis na Costa Rica (Tabela 3), e indicam que não ocorreu endogamia nas populações estudadas.

Como o índice de fixação foi praticamente nulo, as estimativas de tamanho efetivo populacional foram próximas ao número de indivíduos amostrados (Tabela 4). Estes dados mostram que a espécie apresenta alta taxa de polinização cruzada, confirmada neste estudo pelas altas taxas de cruzamento aparente, observadas para ambas as populações $\left(t_{a}=1,11\right.$, Porto Acre e $t_{a}=0,88$, Rio Branco), congruentes com estudos sobre sistema reprodutivo realizados por Cloutier et al. (2007) no Pará, e Hall et al. (1994) na Costa Rica. Ambos verificaram taxa de polinização cruzada multilocos próxima a 1, para esta espécie. Estudos sobre a biologia reprodutiva de C. guianensis indicam a existência de mecanismos de inibição da germinação do pólen na superfície do estigma, em situação de autopolinização, o que a torna obrigatoriamente alógama (Maués, 2006).

O entendimento da relação entre o tamanho efetivo e o tamanho real de uma população é de fundamental 
importância para o planejamento de estratégias de conservação. Segundo Loveless \& Hamrick (1984), quando existe equilíbrio entre os eventos demográficos tais como flutuações temporais no tamanho da população, presença de estruturação e endogamia dentro das populações, diferenças na fertilidade, assincronismo no florescimento, entre outros, os valores de $\hat{\mathrm{N}}_{\mathrm{e}}$ são muito próximos ao número de indivíduos coletados. Foi verificado, por meio da análise comparativa dos dados encontrados neste estudo, com os de outros autores (Tabela 3), que existe uma tendência de os valores estimados de $\hat{\mathrm{N}}_{\mathrm{e}}$ serem próximos ao número de indivíduos amostrados, o que leva a concluir que as populações dessa espécie apresentam equilíbrio nos mecanismos de manutenção da diversidade. Somente no estudo de Kageyama et al. (2004), foram observados valores elevados de endogamia, que resultaram em valor de $\hat{\mathrm{N}}_{\mathrm{e}}$ menor do que o número de indivíduos amostrados. $\mathrm{O}$ aumento da endogamia pode ter sido decorrente da ocorrência de cruzamentos entre indivíduos aparentados, nessas populações, uma vez que a espécie é autoincompatível e, também, pela pequena quantidade de locos analisados.

A diversidade gênica média $\left(\hat{\mathrm{H}}_{\mathrm{e}}=0,606\right)($ Tabela 1$)$ foi menor que a encontrada nos demais estudos com esta espécie (Tabela 3). As duas populações de andiroba de Porto Acre, avaliadas por Kageyama et al. (2004), com apenas dois locos SSR, exibiram diversidade gênica maior $\left(\hat{H}_{\mathrm{e}}=0,74\right)$. Resultados semelhantes a estes também foram obtidos por Cloutier et al. (2007), com seis locos $\left(\hat{H}_{\mathrm{e}}=0,71\right)$, em uma população de andiroba na Floresta Nacional do Tapajós, no Pará. Esta variação encontrada deve estar relacionada, provavelmente, com o número de indivíduos analisados, já que os dois locos analisados por Kageyama et al. (2004) e os seis utilizados por Cloutier et al. (2007) também foram analisados no presente estudo.
A diversidade genética máxima $\left(\hat{\mathrm{H}}_{\max }\right)$ representa o valor máximo teoricamente esperado da diversidade gênica $\left(\hat{H}_{\mathrm{e}}\right)$, de acordo com o número observado de alelos. No presente estudo, os marcadores SSR utilizados detectaram cerca de $70,7 \%$ da diversidade máxima possível (Tabela 1). Esse valor é inferior ao observado nos demais estudos com essa espécie (Tabela 3), mesmo naqueles em que o número de locos utilizados foi reduzido (e.g., Kageyama et al. (2004), com apenas dois locos). Considerando-se o modo como os parâmetros $\hat{\mathrm{H}}_{\mathrm{e}}$ e $\hat{\mathrm{H}}_{\max }$ são calculados, constata-se que a ocorrência de muitos alelos raros em uma população ocasiona um aumento proporcionalmente maior na estimativa de $\hat{\mathrm{H}}_{\text {max }}$, que considera apenas o número observado de alelos, em comparação a $\hat{H}_{\mathrm{e}}$, que considera freqüências alélicas. Portanto, se uma população apresentar valores de $\hat{H}_{\max }$ e $\hat{H}_{\mathrm{e}}$ próximos, provavelmente, não deve possuir muitos alelos em baixa frequiência. Todavia, se ela apresentar valores elevados de $\hat{\mathrm{H}}_{\max }$ e valores relativamente mais baixos de $\hat{\mathrm{H}}_{\mathrm{e}}$, conseqüentemente, ela terá menor porcentagem da máxima diversidade possível, o que indica que esta população apresenta maior número de alelos em baixa freqüência (alelos raros).

Em todos os locos, o número de alelos (Â) foi maior que o número efetivo de alelos ( $\hat{\mathrm{A}}_{\mathrm{e}}$ ) (Tabela 1$)$, o que confirma a distribuição desigual de freqüências alélicas, com número elevado de alelos com baixa freqüência, o que é normal em análises com SSR (Tabela 2). A presença destes alelos explica o fato de a população estudada apresentar valores estimados de $\hat{H}_{\mathrm{e}}$ inferiores aos valores encontrados em outras populações desta mesma espécie (Tabela 3).

O estudo de Cloutier et al. (2007), por exemplo, foi realizado com número de locos semelhante ao do presente estudo e, apesar de a amostragem ter sido cerca de

Tabela 3. Estimativas: $n$ é o número de indivíduos analisados; Âé o número médio de alelos por loco; $\hat{H}_{\mathrm{e}}$ é a heterozigosidade esperada, sob equilíbrio de Hardy-Weinberg; $\hat{\mathrm{H}}_{\max }$ é a máxima diversidade; $\hat{\mathrm{H}}_{\mathrm{e}} / \hat{\mathrm{H}}_{\max }$ é a proporção da diversidade máxima (em

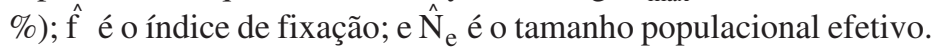

\begin{tabular}{lccccccccc}
\hline Autor & Local & $\mathrm{N}^{\mathrm{o}}$ de locos SSR & $\mathrm{n}$ & $\hat{\mathrm{A}}$ & $\hat{\mathrm{H}}_{\mathrm{e}}$ & $\hat{\mathrm{H}}_{\max }^{(1)}$ & $\hat{\mathrm{H}}_{\mathrm{e}} / \hat{\mathrm{H}}_{\max }$ & $\hat{\mathrm{f}}$ & $\hat{\mathrm{N}}_{\mathrm{e}}$ \\
\hline Dayanandan et al. (1999) & Costa Rica & 3 & 170 & 8,17 & 0,640 & 0,877 & 72,80 & $-0,030^{\mathrm{ns}}$ & 175,25 \\
Kageyama et al. (2004) & Porto Acre - AC & 2 & 133 & 7,87 & 0,740 & 0,873 & 84,80 & $0,251^{*}$ & 106,31 \\
Cloutier et al. (2007) & Flona Tapajós - PA & 6 & 365 & 7,87 & 0,710 & 0,873 & 81,30 & $0,052^{* *}$ & 346,95 \\
O presente trabalho & Acre & 7 & 77 & 7,3 & 0,606 & 0,863 & 70,70 & $0,006^{\text {ns }}$ & 80,71 \\
\hline
\end{tabular}

${ }^{(1)} \hat{\mathrm{H}}_{\max }$ : máxima diversidade de acordo com o número de alelos, estimado a partir do número médio de alelos por loco (A) obtido dos trabalhos. ns Não-significativo. * e **Significativo a 5 e $1 \%$ de probabilidade, respectivamente. 
cinco vezes maior, os autores observaram número médio de alelos similar ao do presente trabalho e estimativas de $\hat{\mathrm{H}}_{\mathrm{e}}$ ligeiramente superiores, que atingiram mais de $81 \%$ da diversidade máxima possível. No presente estudo, provavelmente foi amostrada grande parte da riqueza alélica da espécie, mas como o número de indivíduos amostrados foi reduzido, muitos alelos apresentaram-se em baixa frequiência, o que não deve ter ocorrido no estudo de Cloutier et al. (2007), em que o número de indivíduos analisados foi maior. Essa é uma provável explicação para a população de Tapajós, no Pará, apresentar menor proporção de alelos raros que as populações amostradas no presente estudo. $\mathrm{O}$ mesmo pode ser constatado no estudo de Kageyama et al. (2004), em que há indícios de menor proporção de alelos raros.

Inicialmente, poder-se-ia considerar um contra-senso o fato de que duas populações amostradas em Porto Acre (Kageyama et al. (2004) e o presente estudo) apresentem resultados diferentes, no que se refere à proporção de alelos raros. Entretanto, esta diferença pode estar associada ao menor número de locos utilizados por Kageyama et al. (2004), ao número de indivíduos analisados e, também, ao uso de técnicas diferentes de

Tabela 4. Estimativas de índices de diversidade genética, obtidas para as populações Porto Acre e Rio Branco de Carapa guianensis, em que: n é o número médio de indivíduos analisados; $\hat{\mathrm{A}}_{\mathrm{T}}$ é o número total de alelos; $\hat{A}$ é o número médio de alelos por loco; $\hat{\mathrm{A}}_{\mathrm{e}}$ é o número efetivo de alelos por loco; $\hat{\mathrm{A}}_{\mathrm{p}}$ é o número de alelos exclusivos; $\hat{\mathrm{A}}_{\mathrm{r}}$ é o número de alelos raros; $\hat{H}_{\mathrm{o}}$ é a heterozigosidade observada média; $\hat{H}_{\mathrm{e}}$ é a diversidade gênica; $\hat{f}$ é o índice de fixação; $\hat{\mathrm{t}}_{\mathrm{a}}$ é a taxa de cruzamento aparente; $\hat{\mathrm{N}}_{\mathrm{e}}$ é o tamanho efetivopopulacional; $\hat{\mathrm{N}}_{\mathrm{e}} / \mathrm{n}$ é a relação entre o tamanho efetivo e o tamanho amostral.

\begin{tabular}{ccc}
\hline Parâmetros & Porto Acre & Rio Branco \\
\hline $\mathrm{n}$ & 38,8 & 38,0 \\
$\hat{\mathrm{A}}_{\mathrm{T}}$ & 40 & 38 \\
$\hat{\mathrm{A}}$ & $5,714(0,644)^{(1)}$ & $5,143(0,800)^{(1)}$ \\
$\hat{\mathrm{A}}_{\mathrm{e}}$ & 2,41 & 2,37 \\
$\hat{\mathrm{A}}_{\mathrm{p}}$ & 13 & 11 \\
$\hat{\mathrm{A}}_{\mathrm{r}}$ & 17 & 12 \\
$\hat{\mathrm{H}}_{\mathrm{o}}$ & $0,615(0,100)^{(1)}$ & $0,541(0,081)^{(1)}$ \\
$\hat{\mathrm{H}}_{\mathrm{e}}$ & $0,585(0,085)^{(1)}$ & $0,578(0,088)^{(1)}$ \\
$\hat{\mathrm{f}}\left(\mathrm{IC} \mathrm{C}_{99 \%}\right)^{2}$ & $-0,051(-0,186 \mathrm{a} 0,072)^{(2)}$ & $0,064(-0,093 \mathrm{a} 0,1839)^{(2)}$ \\
$\hat{\mathrm{t}}_{\mathrm{a}}$ & 1,11 & 0,88 \\
$\hat{\mathrm{N}}_{\mathrm{e}}$ & 41 & 36 \\
$\hat{\mathrm{N}}_{\mathrm{e}} / \mathrm{n}$ & 1,05 & 0,94 \\
\hline
\end{tabular}

(1)Erros-padrão. (2)(IC): intervalo de confiança a 99\% de probabilidade, com 10 mil reamostragens bootstrap sobre os locos. detecção dos alelos, pois, no presente trabalho, foi utilizado um analisador automático de fragmentos de DNA, que é mais sensível que a detecção por meio da coloração por nitrato de prata, utilizada por Kageyama et al. (2004).

Pela comparação entre as estimativas de $\hat{H}_{\max }$ e $\hat{H}_{\mathrm{e}}$, constata-se que as populações estudadas por Cloutier et al. (2007) e Kageyama et al. (2004) apresentam menor quantidade de alelos raros, se comparadas com as populações do presente estudo e as de Dayanandan et al. (1999) (Tabela 3), já que estas apresentam valores elevados de $\hat{\mathrm{H}}_{\text {max }}$ e comparativamente menores de $\hat{\mathrm{H}}_{\mathrm{e}}$.

Dessa forma, a comparação entre $\hat{H}_{\max }$ e $\hat{H}_{\mathrm{e}}$ permite averiguar indiretamente a ocorrência de alelos raros nas populações, pois quanto mais próximos forem os valores estimados destes dois parâmetros, menor a quantidade de alelos em baixa freqüência que a população irá apresentar. Constatou-se que, embora as populações de andiroba, já estudadas com marcadores SSR, tenham apresentado níveis consideráveis de diversidade genética, a maior proporção de alelos raros, nas populações do presente estudo e da Costa Rica, indica que essas podem ser mais sensíveis à perda de alelos em conseqüência de deriva genética.

A divergência genética entre as populações foi intermediária $\left(\mathrm{R}_{\mathrm{ST}}=0,093\right)$, o que já era esperado pelo fato de serem encontrados alguns alelos exclusivos nas duas populações, com frequiências relativamente altas (Tabela 2), e também pela distância geográfica entre as duas. Seoane et al. (2000) citam que a presença de alelos exclusivos em algumas populações é indicativa de fluxo gênico restrito, o que pode levar a um aumento da divergência genética entre populações. Embora o fluxo gênico aparente $(\mathrm{Nm}=2,44)$ tenha sido elevado, ele se refere ao fluxo que ocorreu no passado (fluxo gênico histórico), quando as duas populações faziam parte de uma única grande população. Portanto, isto deve ser interpretado com cautela, pois indica que as populações de diferentes regiões, apesar de geograficamente isoladas, apresentam alelos e freqüências similares, como resultado de trocas gênicas antigas, ou seja, até um passado recente, as duas populações constituíam uma metapopulação e, atualmente, existe indício do processo de isolamento pela distância, já que, pela hidrografia da região e pela distância entre as duas populações, o fluxo gênico deve ser restrito. 
Os níveis consideráveis de diversidade genética ( $\hat{\mathrm{A}}$ e $\hat{\mathrm{H}}_{\mathrm{e}}$ ), observados em diferentes populações, e a ausência de endogamia, decorrente de mecanismos reprodutivos de incompatibilidade, são características indicativas de manutenção em longo prazo da diversidade genética. Essa constatação é corroborada, ainda, pela representatividade genética $\left(\hat{\mathrm{N}}_{\mathrm{e}}\right)$ das populações. Entretanto, como já comentado, apenas esses parâmetros são insuficientes para atestar o status de conservação em longo prazo da espécie. Observou-se, por meio comparação das estimativas de $\hat{\mathrm{A}}, \hat{\mathrm{H}}_{\mathrm{e}}$ e $\hat{\mathrm{H}}_{\max }$, que a proporção de alelos raros difere entre as populações, e que as populações com maior proporção de alelos raros podem ser mais sensíveis à perda de diversidade genética decorrente da deriva genética.

\section{Conclusões}

1. As duas populações de andiroba do Acre têm índices elevados e similares de diversidade genética, e a divergência genética entre as duas populações é intermediária.

2. A comparação entre $\hat{H}_{\max }$ e $\hat{H}_{\mathrm{e}}$ permite averiguar, indiretamente, a ocorrência de alelos raros nas populações.

3. As populações dessa espécie apresentam equilíbrio nos mecanismos de manutenção da diversidade.

4. Níveis consideráveis de diversidade genética ( $\hat{\mathrm{A}}$ e $\hat{\mathrm{H}}_{\mathrm{e}}$ ) em diferentes populações, ausência de endogamia e os valores de representatividade genética $\left(\hat{\mathrm{N}}_{\mathrm{e}}\right)$ são características indicativas de manutenção em longo prazo da diversidade genética, porém insuficientes para atestar o status de conservação da espécie.

5. Para definir estratégias de conservação in situ, deve ser realizada uma análise complementar do número de alelos de baixa freqüência ou de parâmetros que indicam indiretamente a ocorrência desses alelos, como o número efetivo de alelos por loco $\left(\hat{\mathrm{A}}_{e}\right)$ e a comparação entre $\hat{\mathrm{H}}_{\mathrm{e}}$ e $\hat{\mathrm{H}}_{\max }$.

\section{Agradecimentos}

Ao CNPq, pela concessão de bolsas de produtividade científica; ao Instituto Internacional de Educação do Brasil, pelo apoio financeiro; à Embrapa Acre, pelo auxílio às viagens de coleta; à Embrapa
Recursos Genéticos e Biotecnologia, Laboratório de Genética Vegetal, pelo apoio técnico e material para realização deste trabalho; a Aldeci da Silva Oliveira, da Embrapa Acre, pela colaboração nas coletas.

\section{Referências}

APPLIED BIOSYSTEMS. Genescan 3.7. Foster City: Applied Biosystems, 2001. CD-ROM.

APPLIED BIOSYSTEMS. Genotyper 2.0. Foster City: Applied Biosystems, 1996. CD-ROM.

BERG, E.E.; HAMRICK, J.L. Quantification of genetic diversity at allozyme loci. Canadian Journal of Forest Research, v.27, p.415424, 1997.

BRONDANI, R.P.V.; GRATTAPAGLIA, D. A simple and cost effective method to synthesize a fluorescent labeled internal DNA standard for fragment sizing in an automatic sequencer. Biotechniques, v.31, p.793-800, 2001.

CLOUTIER, D.; KANASHIRO, M.; CIAMPI, A.Y.; SCHOEN, D.J. Impact of selective logging on inbreeding and gene dispersal in an Amazonian tree population of Carapa guianensis Aubl. Molecular Ecology, v.16, p.797-809, 2007.

CROSSA, J.; VENCOVSKY, R. Implications of the variance effective population size on the genetic conservation of monoecious species. Theoretical and Applied Genetics, v.89, p.936-942, 1994.

DAYANANDAN, S.; DOLE, J.; BAWA, K.; KESSELE, R. Population structure delineated with microsatellite markers in fragmented populations of a tropical tree, Carapa guianensis (Meliaceae). Molecular Ecology, v.8, p.1585-1592, 1999.

DOYLE, J.J.; DOYLE, J.L. Isolation of plant DNA from fresh tissue. Focus, v.12, p.13-15, 1990.

GOODMAN, S.J. $\mathrm{R}_{\mathrm{ST}}$ Calc: a collection of computer programs for calculating estimates of genetic differentiation from microsatellite data and determining their significance. Molecular Ecology, v.6, p.881-885, 1997.

GOUDET, J. F $_{\text {STAT }}$ (version 1.2): a computer program to calculate F-statistics. Journal of Heredity, v.86, p.485-486, 1995.

HALL, P.; ORREL, L.C.; BAWA, K. Genetic diversity and mating system in a tropical tree, Carapa guianensis (Meliaceae). American Journal of Botany, v.81, p.1104-1111, 1994.

HAMRICK, J.L.; GODT, M.J.W. Conservation genetics of endemic plant species. In: AVISE, J.C.; HAMRICK, J.L. (Ed.). Conservation genetics: case histories from nature. New York: Chapman \& Hall, 1996. p.281-304.

KAGEYAMA, P.Y.; CARON, D.; GANDARA, F.B.; MARTINS, K.; WADT, L.H.O.; LACERDA, C.M.B.; BOUFLEUER, N.T.; RIBAS, L.A.; MORENO, M. A.; FERRAZ, E.M. Genetic and ecological aspects of nonwood forest product exploitation in two Western Amazonian settlements. In: VINCENTI, B.; AMARAL, W.; MEILLEUR, B. (Org.). Challenges in managing forest genetic resource for livelihoods: examples from Argentina and Brazil. Roma: IPGRI, 2004, p.149-217. 
LANDE, R. Genetic and demography in biological conservation. Science, v.241, p.1455-1460, 1988.

LEWIS, P.; ZAYKIN, D. Genetic data analysis: computer programe for the analyses of allelic data - version 1.0 (d2). Alburquerque: University of New Mexico, 2002. Disponível em: <http:// alleun.eeb.uconn.edu/gda>. Acesso em: 20 nov. 2003.

LOVELESS, M.D.; HAMRICK, J.L. Ecological determinants of genetic structure in plant populations. Annual Review of Ecology and Systematics, v.15, p.65-95, 1984.

MAUÉS, M.M. Estratégias reprodutivas de espécies arbóreas e a sua importância para o manejo e conservação florestal: Floresta Nacional do Tapajós (Belterra-PA). 2006. 206p. Tese (Doutorado) Universidade de Brasília, Brasília.

SEOANE, C.E.S.; KAGEYAMA, P.Y.; SEBBEN, A.M. Efeitos da fragmentação florestal na estrutura genética de populações de Esenbeckia leiocarpa Engl. (Guarantã). Scientia Forestalis, v.57, p.123-139, 2000.

SLATKIN, M. A measure of population subdivision based on microsatellite allele frequencies. Genetics, v.139, p.457-462, 1995.
VENCOVSKY, R. Variance of an estimate of the outcrossing rate. Brazilian Journal of Genetics, v.17, p.349-351, 1994.

VENCOVSKY, R.; CROSSA, J. Measurements of representativeness used in genetic resources conservation and plant breeding. Crop Science, v.43, p.1912-1921, 2003.

VINSON, C.C.; AZEVEDO, V.C.R.; SAMPAIO, I.; CIAMPI, A.Y. Development of microsatellite markers for Carapa guianensis (Aublet), a tree species from the Amazon Forest. Molecular Ecology Notes, v.5, p.33-34, 2005.

WEIR, B.S. Genetic data analysis II: methods for discrete population genetic data. Sunderland: Sinauer Associates, 1996. 445p.

WEIR, B.S.; COCKERHAM, C.C. Estimating F-statistics for the analysis of population structure. Evolution, v.38, p.1358-1370, 1984.

WRIGHT, S. The genetical structure of populations. Annals of Eugenics, v.15, p.323-354, 1951.

YOUNG, A.; BOYLE, T.; BROWN, T. The population genetic consequences of habitat fragmentation for plants. Trends in Ecology and Evolution, v.11, p.413-418, 1996. 